\title{
Weighted Network of Chinese Nature Science Basic Research
}

\author{
Jian-Guo Liu ${ }^{1 *}$, Zhao-Guo Xuan ${ }^{1}$, Yan-Zhong Dang ${ }^{1}$, Qiang Guo ${ }^{2}$, and Zhong-Tuo Wang ${ }^{1}$ \\ ${ }^{1}$ Institute of System Engineering, Dalian University of Technology, Dalian 116023, $P$ R China \\ ${ }^{2}$ School of Science, Dalian Nationalities University, Dalian 116600, $P R$ China
}

\begin{abstract}
Using the requisition papers of Chinese Nature Science Basic Research in management and information department, we construct the weighted network of research areas(WRAN) represented by the subject codes. In WRAN, two research areas are considered connected if they have been filled in at least one requisition paper. The edge weight is defined as the number of requisition papers which have filled in the same pairs of codes. The node strength is defined as the number of requisition papers which have filled in this code, including the papers which have filled in it only. Here we study a variety of nonlocal statistics for these networks, such as typical distances between research areas through the network, and measures of centrality such as betweenness. These statistics characteristics can illuminate the global development trend of Chinese scientific study, it is also helpful to adjust the code system to reflect the real status more accurately. Finally, we present a plausible model for the formation and structure of networks with the observed properties.
\end{abstract}

PACS numbers: $89.75 . \mathrm{Hc} ;$ 89.75.Da

\section{INTRODUCTION}

Recently, the topological properties and evolutionary processes of complex networks are used to describe the relationships and collective behaviors in many fields [1, 2, 3, 4, 5, 6, 7]. Some new analysis methods and topology properties have been proposed by network analysis. Also it impelled us to study the complex system from the point of macroscopically view. A network is consisted of a set of nodes and edges which represent the relationship between any two nodes. The topological network is denoted by an adjacent matrix $W=w_{i j}$, if node $i$ connect to node $j, w_{i j}=1$; Otherwise, $w_{i j}=0$. Just because of its simplicity of this description, network can be used in so many different subjects, such as collaboration of scientists [8, 9, 10, 11], Internet networks 12], World-Wide Web 2], the collaborative research and project bipartite network 13] and so on. Barber et. al 13] studied the collaboration network consisting of research projects funded by the European Union and the organizations. They found that the collaboration network has the main characteristics, such as scale-free degree distribution, small average distance, high clustering and assortative node correlations. However, the real systems are far from Boolean structure. The purely topological characterization will miss important attributes often encountered in real systems. So to fully characterize the interactions in real-world networks, weight of links should be taken into account. In fact, there are already many works on weighted networks, including empirical studies 14, 15, 16, 17, 18, 19] and evolutionary models 18, 20, 21, 22, 23, 24, 25].

The empirical study of weighted network without a naturally given definition of weight is especially valuable to answer questions such as how to define a well behav-

*Electronic address: liujg004@yahoo.com.cn ior weight, and to extract structural information from networks, and what's the role of weight according to its effects on the structure of the network. We introduce some metrics that combine in a natural way both the topology of the connections and the weight assigned to them. These quantities provide a general characterization of the heterogenous statistical properties of weights and identify alternative definitions of centrality, local cohesiveness, and affinity. By appropriate measurements it is also possible to exploit the correlation between the weights and the topological structure of the network, unveiling the complex architecture shown by real weighted networks.

The scientific studies can be considered as being organized within a network structure, which has a significant influence on the observed study collective behaviors. The viewpoints of complex networks are of interest in studying scientific study networks to uncover the structural characteristics of WRAN. The topological statistics properties have discussed in Ref. 26]. In the fund management department, such as National Natural Science Foundation of China (NSFC), the research areas are denoted by the code system, which have the tree structure to demonstrate the inclusion relation between the research areas, such as Physics->statistical physics->complex network. The leave codes of the code system always represent the research areas more specially. To make the network reflect the reality more accurately, the nodes are defined as the codes. Because the scientists can fill in the fund proposal two codes: the first application code and the second one, then if one requisition paper filled in two different codes one can consider that the research work is cross the two research areas. The edge weight $w_{i j}$ between node $i$ and $j$ is defined as the number of papers filled in the two codes. The node strength $s_{i}$ is defined as the number of requisition papers which have filled code $i$, including the papers which have filled it only. By this definition, the network size $N$ is 321 in WRAN from 1999 to 2004 . The network shows all the main characteristics 
known from other complex network structure, such as exponential distribution of degree, node weight and node strength, small average path length, large clustering, and assortative node correlations. Besides the general interest in studying the new network, the study could help us to know how the network structure affects network functions such as knowledge creation, knowledge diffusion and the collaboration of scientists. Moreover, the macroscopically analysis can illuminate the global development trend of Chinese scientific study, it is also helpful to adjust the code system to reflect the real status more accurately.

\section{MEASUREMENT OF WEIGHT AND BASIC STATISTICAL RESULTS}

Now we turn to the effects of weight on the structure of weighted networks. First, the interaction weight $w_{i j}$ is define as the number of requisition papers which have filled in code $i$ and code $j$. The strength $s_{i}$ of node $i$ is defined as

$$
s_{i}=\sum_{j \in \Gamma_{i}} w_{i j}+\eta_{i}
$$

where $\Gamma_{i}$ is the neighbor node set of node $i$ and the fitness $\eta_{i}$ is the number of requisition papers which filled in the code $i$ only. The weight $w_{i}$ of node $i$ is defined as

$$
w_{i}=\sum_{j \in \Gamma_{i}} w_{i j}
$$

This quantity measures the strength of nodes in terms of the total weight of their connections. The distributions of degree, node weight and node strength are demonstrated in Fig 1 The probability distribution $P(s)$ that a node has strength $s$ is exponential distribution, and the functional behavior exhibits similarities with the degree distribution $P(k)$ (see Fig 1). The largest strength nodes have been listed in Table 1 .

A precise functional description of the exponential distributions may be very important for understanding the network evolution and will be deferred to future analysis. To shed more light on the relationship between the node strength and degree, we investigate the dependence of $s_{i}$ on $k_{i}$. We find that the average strength $s(k)$ and weight $w(k)$ of nodes with degree $k$ increase with the degree as

$$
s(k) \sim k^{\beta_{s k}}, \quad w(k) \sim k^{\beta_{w k}} .
$$

The real data follows the power-law behavior with exponent $\beta_{s k}=1.14 \pm 0.02$ and $\beta_{w k}=1.12 \pm 0.01$ (see Fig 21). The two exponents denote anomalous correlations between the number of paper which has filled in one node and the number of its connections, and imply that the strength and weight of nodes grows faster than their degree and the weight of edges belonging to highly connected nodes tends to have a higher value. This tendency denotes a strong correlation between the strength, node weight and the topological properties in WRAN. The difference between $\beta_{s k}$ and $\beta_{w k}$ implies that the larger degree a node is, the more fitness $\eta_{i}$ it has.

Table 1, The hub nodes of WRAN and their strength from 1999 to 2004.

\begin{tabular}{lcc}
\hline Year & Hub nodes & $s$ \\
\hline 1999 & Corporation theory & 178 \\
2000 & Macroscopical economy management and stratagem & 79 \\
2001 & Corporation stratagem management & 93 \\
2002 & Computer network, distributed computer system(CNDCS) & 83 \\
2003 & CNDCS & 132 \\
2004 & CNDCS & 194 \\
\hline
\end{tabular}

\section{A. Distance and Centrality}

Shortest path play an important role in the transport and communication within a network, it have also played an important role in the characterization of the internal structure of a network 27, 28]. The average distance, denoted by $D=\frac{1}{N(N-1)} \sum_{i j} d_{i j}$, represent all the average shortest path lengths of a network in which the entry $d_{i j}$ is the shortest path length from node $i$ to node $j$. It should be noticed that all the network nodes are not all connected in the six years. The largest connected group has 256, 279, 293, 290, 309 and 310 nodes, respectively. The average distance is discussed on the largest connected group. The ability of two nodes, $i$ and $j$, to communicate with each other depends on the length of the shortest path $d_{i j}$ between them. The average distance from node $i$ to all other nodes is defined as

$$
D_{i}=\frac{1}{N-1} \sum_{j=1, j \neq i}^{N} d_{i j} .
$$

In the Boolean structure network, if nodes $i$ and $j$ are connected, $d_{i j}=1$. In WRAN, the larger edge weight $w_{i j}$ is, the closer relationship between the two nodes have. Thus, the weighted distance $d_{i j}$ is taken $d_{i j}=1 / w_{i j}$. The weighted shortest path length $d_{i j}$ of WRAN is de- 


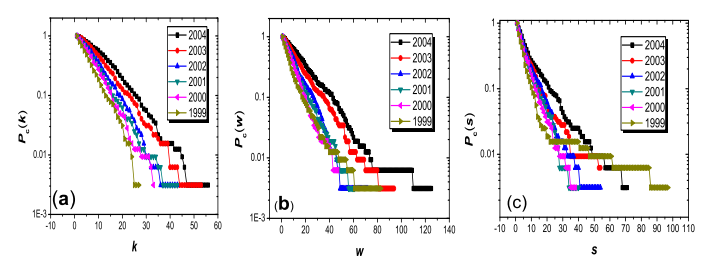

FIG. 1: (Color online) Characteristics of WRAN, such as the distributions of degree, node weight and node strength.

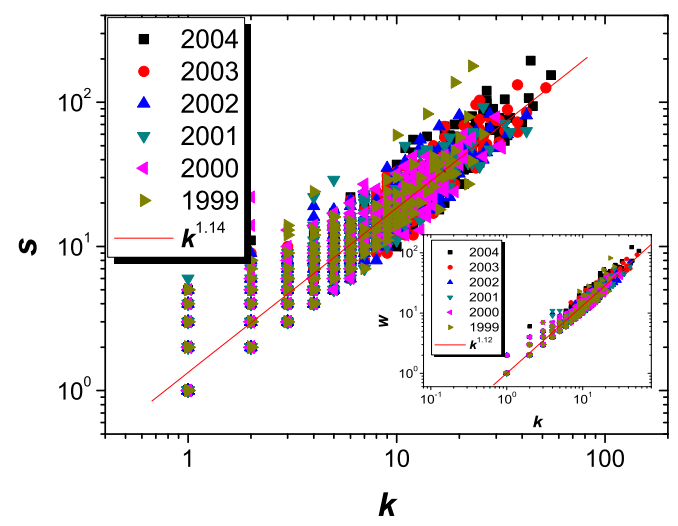

FIG. 2: (Color online) Average strength $s(k)$ as function of the degree $k$ of nodes from 1999 to 2004. The inset figure shows the relationship between the average node weight $w(k)$ and the degree $k$.

fined as the smallest sum of the distance throughout all the possible paths in the network from node $i$ to $j$. Figure 3 4 demonstrate the topological and weighted $D_{i}$ distributions from 1999 to 2004 respectively, which both obey Passion distribution. From the two figures, we can obtain that most nodes' average distance $D_{i}$ are around 3.5 and 2.2 in topological and weighted network, respectively. The nodes belonging to the left part of Passion distribution are very important to the network, because their average distance to all other nodes is very small. The two inset figures show that the average distance $D$ of topological and weighted network decreases with time. This may caused by the increase of the average degree $\langle k\rangle$ (See Fig. 5). Since the number of requisition papers $E$ can be obtained from the equation $E=N\langle k\rangle$ approximately, the real reason why the average distance decrease may lie in the increasing number of requisition papers.

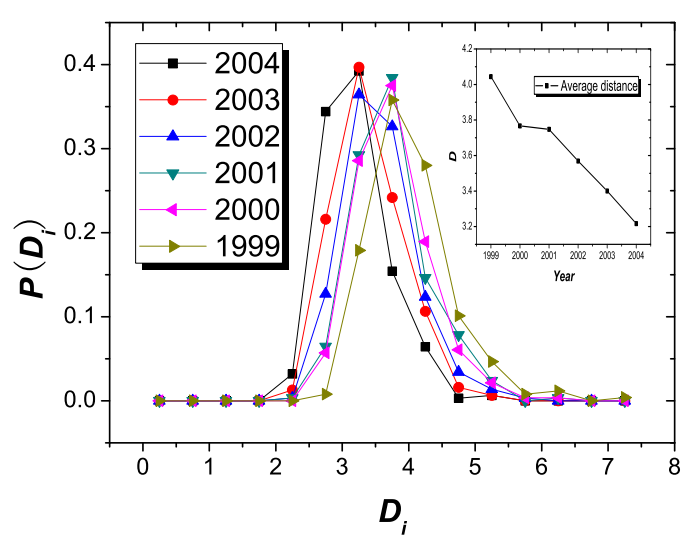

FIG. 3: (Color online) The topological $D_{i}$ distributions from 1999 to 2004 obey Passion distribution. The inset figure shows the average distance $D$ from 1999 to 2004 .

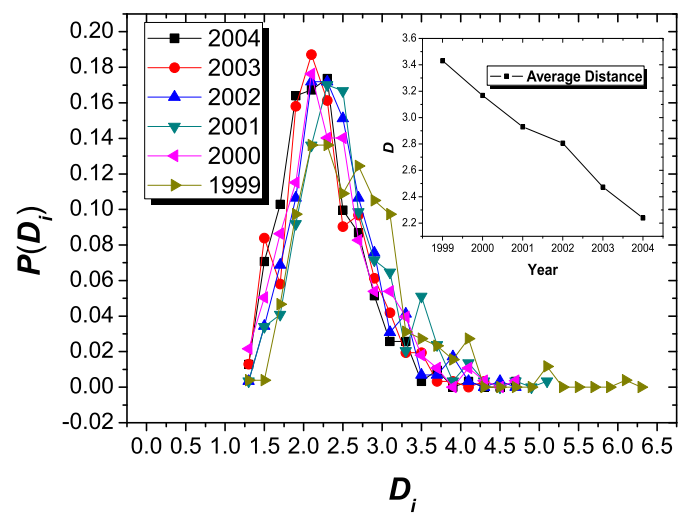

FIG. 4: (Color online) The weighted $D_{i}$ distributions of WRAN from 1999 to 2004 obey Passion distribution. The inset figure shows the average distance $D$ of weighted RAN from 1999 to 2004.

\section{B. Average Clustering coefficient}

The local clustering coefficient of node $i$, denoted by $C_{i}$, is a measure of the connectedness between the neighbors of the node, which is called transitivity in the social network [1, 27]. If a node $i$ has a link to node $j$ and node $j$ has a link to node $k$, then a measure of transitivity in the network is the probability that node $i$ has a link to node $k$. Let $k_{i}$ denote the degree of node $i$, and let $E_{i}$ denote the number of link between the $k_{i}$ neighbors. Then, for an undirected network, the quantity [1]

$$
C_{i}=\frac{2 E_{i}}{k_{i}\left(k_{i}-1\right)}
$$




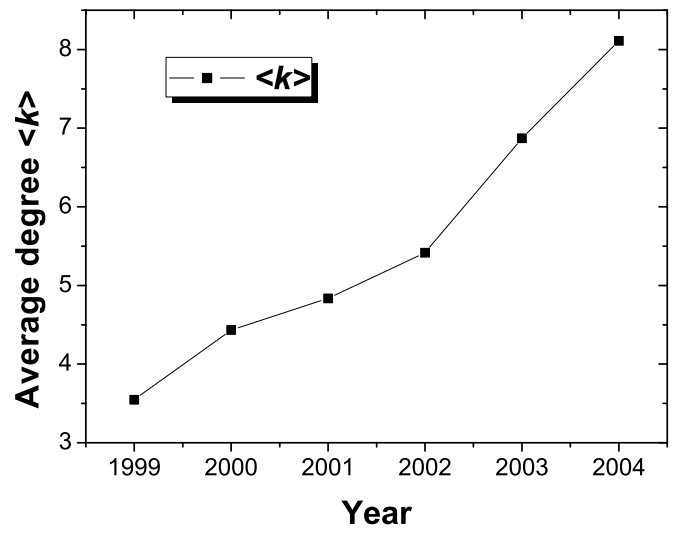

FIG. 5: (Color online) Average degree $\langle k\rangle$ from 1999 to 2004, which is increase almost 2 times from 1999 to 2004.

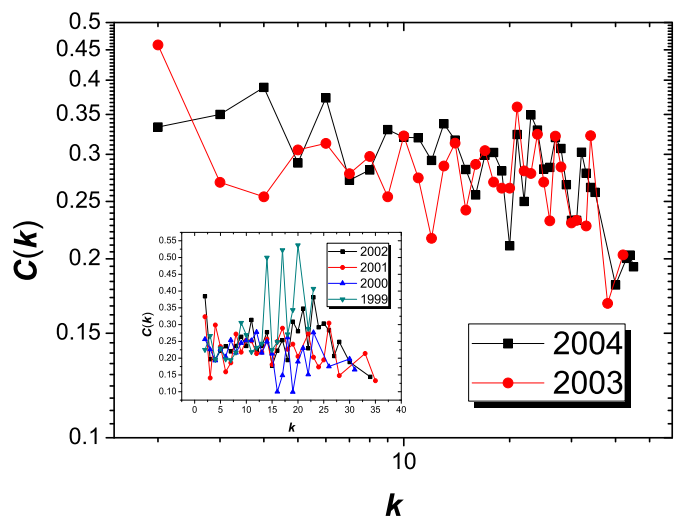

FIG. 6: (Color online)The topological clustering coefficient vs time from 1999 to 2004.

is the ratio of the number of links between a node's neighbors to the number of links that can exist. The clustering coefficient $C$ is defined as $C=1 / N \sum_{i=1}^{N} C_{i}$. In WRAN, the clustering coefficient indicates the probability that a node connects to its 2 nd nearest neighbors. Figure 6 presents the statistic result of $C(k) \sim k$. From Fig 6 we can obtain that there are no correlation between $C(k)$ and $k$ before 2003, but the correlation emerged since 2003, which is a characteristic of hierarchical network. The reason may lie in the fact that the code system has been adjusted around 2002. This result indicates that the rectification make the relationship of the subject codes becoming more clear.

The weighted clustering coefficient is defined as

$$
c_{i}^{w}=\frac{1}{s_{i}\left(k_{i}-1\right)} \sum_{j, h \in \Gamma_{i}} \frac{w_{i j}+w_{i h}}{2} a_{i j} a_{i h} a_{j h} .
$$

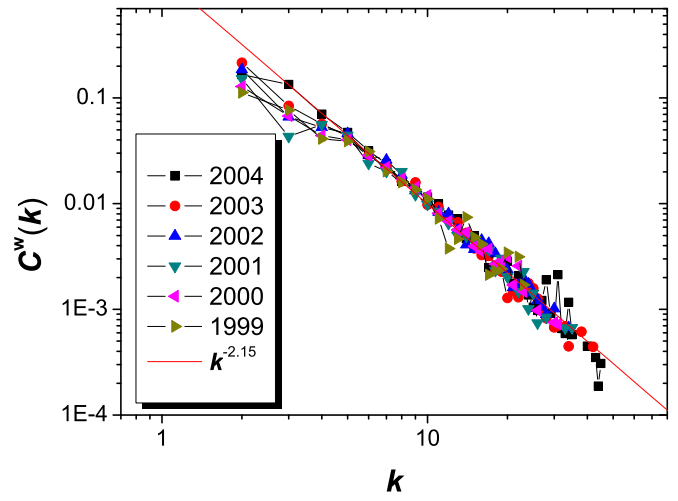

FIG. 7: (Color online)The weighted clustering coefficient vs time from 1999 to 2004.

This coefficient is a measure of the local cohesiveness that takes into account the importance of the clustered structure on the basis of the amount of traffic or interaction intensity actually found on the local triplets. Indeed, $c_{i}^{w}$ counts for each triplet formed in the neighborhood of the node $i$ the weight of the two participating edges of the node $i$. In this way we are considering not just the number of closed triplets in the neighborhood of a node but also their total relative weight with respect to the strength of the node. Consistently, the $c_{i}^{w}$ definition recovers the topological clustering coefficient in the case that $w_{i j}$ is constant and $\eta_{i}=0$. Next we define $C^{w}$ and $C^{w}(k)$ as the weighted clustering coefficient averaged over all nodes of the network and over all nodes with degree $k$, respectively. These quantities provide global information on the correlation between weights and topology, especially by comparing them with their topological analogs. Figure 7 presents the power-law correlations $C^{w}(k) \sim k^{\alpha}$ between $C^{w}(k)$ and degree $k$, where $\alpha=-2.15 \pm 0.06$, which may be caused by the introduction of node fitness $\eta_{i}$. Because the larger the degree $k$ is the larger $\eta_{i}$ would have, the denominator of Equ. (6) would become more larger, then $C^{w}(k)$ would become small. If replace $s_{i}$ of Equ. (6) with $k_{i}$, we get the definition of weighted clustering coefficient presented in Ref. 17]. Figure 8 presents the relationship between $C^{w}$ and $C$ of WRAN. The fact $C^{w}<C$ signals a network in which the topological clustering is generated by edges with low weight or by nodes with larger fitness. In this case the clustering has a minor effect in the organization of the network because the largest part of the interactions is occurring on edges not belonging to interconnected triplets. The figure also indicates that $C$ increase with time, while $C^{w}$ keep constant. Interestingly, $C$ increase dramatically about 10 percent from 2002 to 2003 . This change is consistent with the correlation $C(k) \sim k$.

Along with the weighted clustering coefficient, we introduce the weighted average nearest-neighbors 


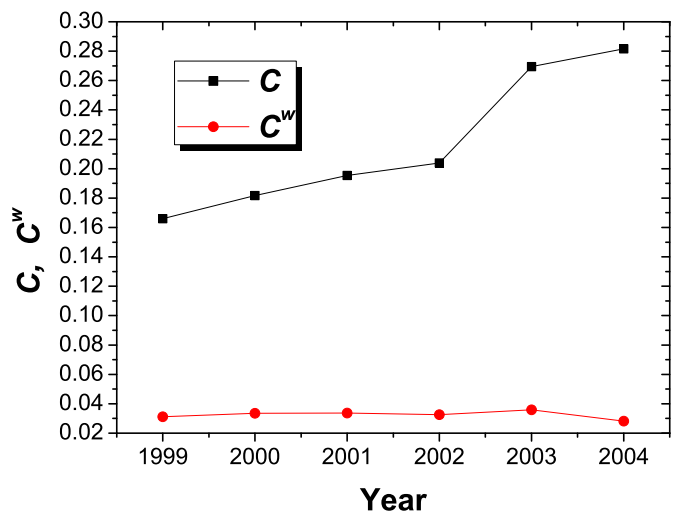

FIG. 8: (Color online) Topological and weighted clustering coefficient of WRAN from 1999 to 2004.

degree [17], defined as

$$
k_{n n, i}^{w}=\frac{1}{s_{i}} \sum_{j=1}^{N} a_{i j} w_{i j} k_{j} .
$$

In this case, we perform a local weighted average of the nearest-neighbor degree according to the normalized weight of the connecting edges, $w_{i j} / s_{i}$. This definition implies that if the edges with the larger weight are pointing to the neighbors with larger degree, $k_{n n, i}^{w}>k_{n n, i}$; In the opposite case $k_{n n, i}^{w}<k_{n n, i}$. Thus, $k_{n n, i}^{w}$ measures the effective affinity to connect with high- or lowdegree neighbors according to the magnitude of the actual interactions. Moreover, $k_{n n}^{w}(k)$ marks the weighted assortative or disassortative properties considering the actual interactions among the systems elements. Figure 9 presents the topological and weighted average nearestneighbors degree of 1999 and 2004, which demonstrate that $k_{n n, i}^{w}>k_{n n, i}$ and both of them have the trend of increasing with the degree $k$.

The positive assortative coefficient $r$, which is presented by Ref. [29, 30], of WRAN has presented in Fig[10] which means that the nodes with higher degree would like to connect each other. Figure 2 told us that the nodes, whose degree is large, must have larger strength. Then, the nodes with more strength would like to connect each other.

\section{Betweenness}

The communication of two non-adjacent nodes, called $j$ and $k$, depends on the nodes belonging to the paths connecting $j$ and $k$. Consequently, the definition node betweenness is present to measure the relevance of a given node by counting the number of geodesics going through it. The betweenness is one of the standard measures of

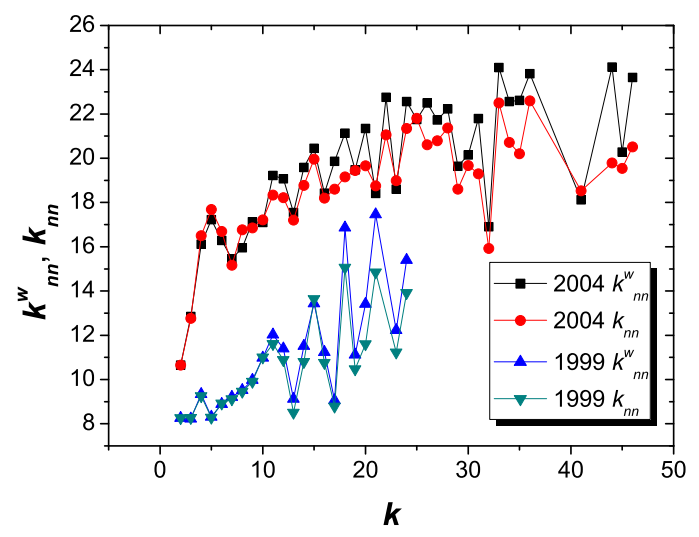

FIG. 9: (Color online)Topological and weighted average nearest-neighbors degree of WRAN of 1999 and 2004.

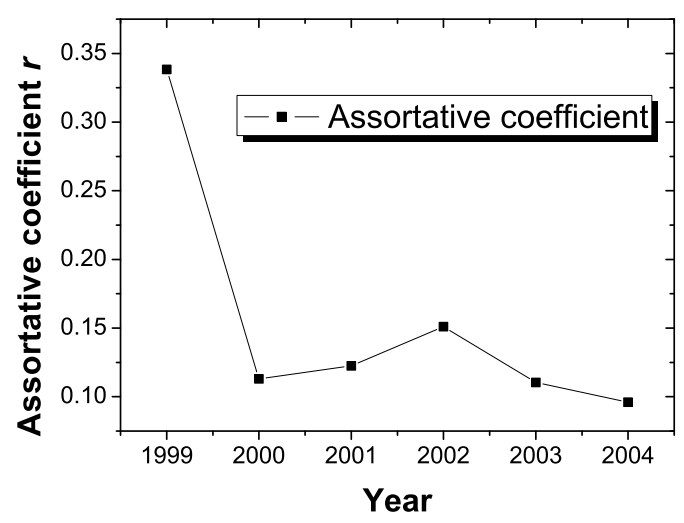

FIG. 10: (Color online)Assortative coefficient vs time of WRAN.

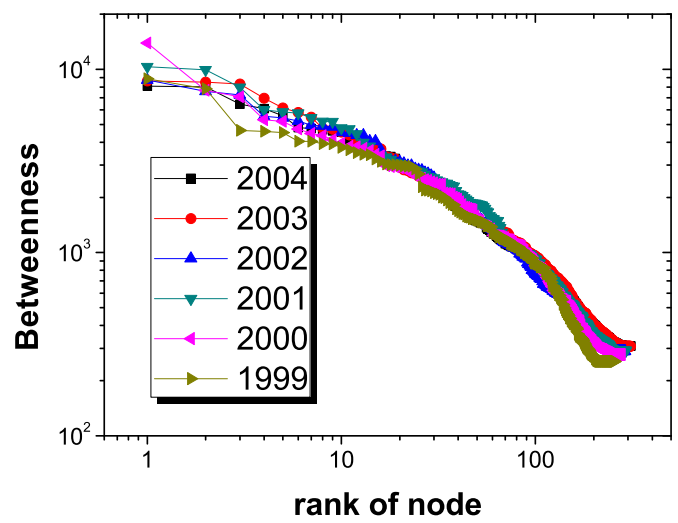

FIG. 11: (Color online)Zipf plots of node betweenness for topological WRAN from 1999 to 2004. 


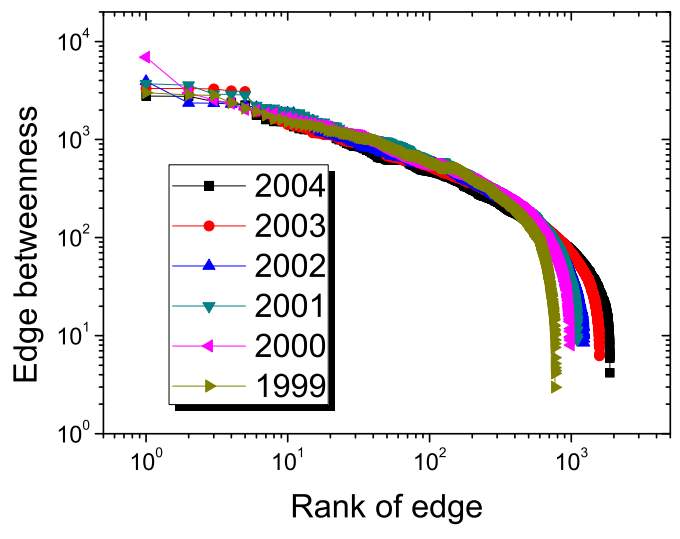

FIG. 12: (Color online)Zipf plots of edge betweenness for topological WRAN from 1999 to 2004.

node centrality. The betweenness $b_{i}$ of node $i$, is defined as 27, 28, 31, 32.

$$
b_{i}=\sum_{j, k=1, j \neq k}^{N} \frac{n_{j k}(i)}{n_{j k}}
$$

where $n_{j k}$ is the number of shortest paths connecting $j$ and $k$, while $n_{j k}(i)$ is the number of shortest paths connecting $j$ and $k$ and passing through $i$. This quantity is an indicator of which node is the most influential one in the network is. The nodes with highest betweenness also result in the largest increase in typical distance between others when they are removed. The nodes with largest betweenness have listed in Table 2. These nodes are the most important one for information transitivity.

The edge betweenness is defined as the number of shortest paths between pairs of nodes that run through that edge [33].

Table 2 The node wich has largest betweenness from 1999 to 2004.

\begin{tabular}{lc}
\hline & The node with largest betweenness \\
\hline 1999 & Computer-aided design \\
2000 & Intelligent information processing \\
2001 & Management information system \\
2002 & Management information system \\
2003 & Artificial intellegence(AI) \\
2004 Intelligent information processing(IIP) \\
\hline
\end{tabular}

\section{A MUTUAL SELECTION MODEL}

In this section, we present a mutual selection model (MSM) to compare with WRAN. Inspired by the fitness $\eta_{i}$ and the mutual selection mechanism, the model is defined as following. The model starts from $N$ isolated nodes, each with an initial attractiveness $s_{0}$. In this paper, $s_{0}$ is set to be 1 . At each time step, every node strength of the network would increase by 1 with the probability $p$; With the probability $(1-p)$, each existing node $i$ selects $m$ other existing nodes for potential interaction according to the probability Equ. (9). Here, the parameter $m$ is the number of candidate nodes for creating or strengthening connections, $p$ is the probability that a node would enhance $\eta_{i}$ by 1 .

$$
\Pi_{i \rightarrow j}=\frac{s_{j}}{\sum_{k(k \neq i)} s_{k}} .
$$

where $s_{i}=\sum_{j \in \Gamma(i)} w_{i j}+\eta_{i}$. If a pair of unlinked nodes is mutually selected, then an new connection will be built between them. If two connected nodes select each other, then their existing connection will be strengthened, i.e., their edge weight will be increased by 1 . We will see that the model can generate the observed properties of WRAN. When $p=0.01$ and $m=5$, the numerical results to different time step $T$ are demonstrated in Fig. 13.??. Figure 13. (a)-(c) give the exponential distributions of degree, node strength and edge weight. Figure 13. (d) demonstrate the power-law relationship between degree $k$ and node strength $s$. Figure 14 demonstrates the increasing trend of $C$, decreasing trend of $D$ and $r$ and the $C^{w}(k) \sim k^{\lambda}$ relationship. From the inset of Fig [14 (b), one can see that when the time step $T$ is very small, there is no correlation between $C(k)$ and $k$, while when $T$ is become large, the correlation emerge, which consistent with $C(k) \sim k$ of WRAN. Figure 14. (b) gives the power-law relationship $C^{w}(k) \sim k^{\alpha}$, where $\alpha=1.11 \pm 0.05$, which also consistent with the one of WRAN. The inset of Figure 14](d) gives the Zipf plots of node betweenness to different time step $T$. All of the above structural characters of MSM are consistent with the ones of WRAN approximately, which indicate that the mutual selection mechanism and the probability $p$ may be the evolving mechanism of WRAN.

\section{CONCLUSIONS AND DISCUSSIONS}

We have studied the Chinese Nature Science Basic Research in management and information department from weighted network point of view. To describe the status of WRAN more accurately, the requisition papers which have filled in only one subject code is also considered, which is defined as node fitness. We have looked at a variety of nonlocal properties of our networks.

Using this measure we have added weighting to WRAN and used the resulting networks to find which code have the largest strength, the shortest average distance to others. Generalization of the clustering coefficient and betweenness calculations to these weighted networks is also straightforward. The statistic characterization give the following conclusions

(1). The code system have adjusted around 2002 and the correlation between $C(k) \sim k$ emerges since 2003 . 

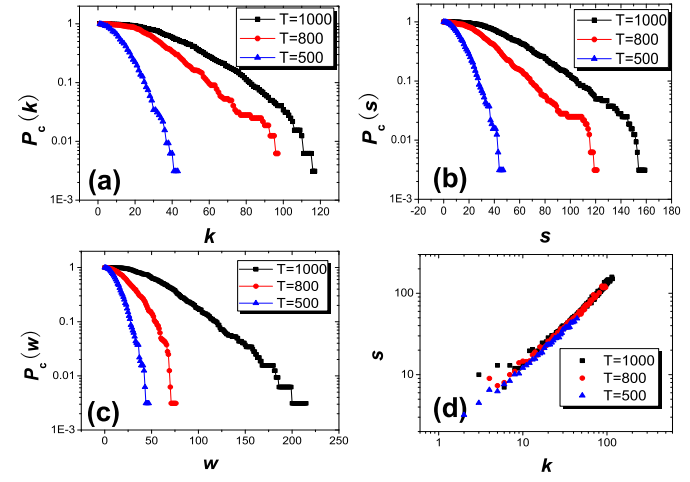

FIG. 13: (Color online) Simulated distributions of degree, node strength and edge weight to different time step $T$. (d) give the relationship between $k$ and $s$.
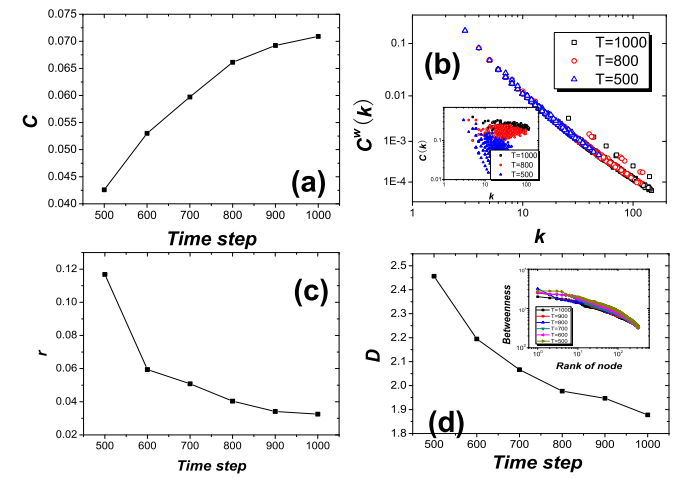

FIG. 14: (Color online) Simulated numerical results of $C, D$ and $r$ to different $T$, and the relationship of $C(k) \sim k$ and $C^{w}(k) \sim k$, which .
(2). The topological and weighted distance decrease with time, while the clustering coefficient increases with time.

(3). The distributions of degree, edge weight and node strength have exponential form.

(4). The larger the node degree is, the larger fitness it would be.

(5). WRAN is assortative, which means that the node with large strength would like to connect each other.

In terms of structural characteristics of WRAN, the present analysis yields a plausible model. Based on the mutual selection mechanism and the probability $p$ that one node would increase its strength without creating new connectivity with others, we presented MSM model. Most of the structural characters of MSN are consistent with the ones of WRAN.

The calculations presented in this paper inevitably represent only a small part of the investigations that could be conducted using large network data sets such as these. We hope, given the high current level of interest in network phenomena, that others will find many further uses for these data.

\section{Acknowledgements}

The authors thank W. -X. Wang and T. Zhou for their valuable comments and suggestions. This work has been partly supported by the Natural Science Foundation of China under Grant Nos. 70431001 and 70271046.
[1] D. J. Watts and S. H. Strogatz, Nature 393, 440 (1998).

[2] A. -L. Barabási and R. Albert, Science 286, 509 (1999).

[3] R. Albert and A. -L. Barabási, Rev. Mod. Phys. 74, 47 (2002).

[4] S. N. Dorogovtsev and J. F. F. Mendes, Adv. Phys. 51, 1079 (2002).

[5] M. E. J. Newmann, SIAM Rev. 45, 167 (2003).

[6] X. F. Wang, Int. J. Bifurcat. Chaos 12, 885 (2002).

[7] S. Boccaletti, V. Latora, Y. Moreno, M. Chavez and D. -U. Hwang, Phys. Rep. 424, 175 (2006).

[8] M. E. J. Newman, Proc. Natl. Acad. Sci. 98, 404 (2001).

[9] M. E. J. Newman, Phys. Rev. E 64, 016131 (2001).

[10] M. E. J. Newman, Phys. Rev. E 64, 016132 (2001).

[11] A. -L. Barabási, H. Jeong, Z. Néda, E. Ravasz, A. Schubert and T. Vicsek, Physica A 311, 590 (2002).

[12] R. Pastor-Satorras and A. Vespignani, Evolution and
Structure of the Internet: A Statistical Physics Approach (Cambridge University Press, Cambridge, England, 2004).

[13] M. J. Barber, A. Krueger, T. Krueger and T. RoedigerSchluga, Phys. Rev. E 73, 036132 (2006).

[14] R. Guimera and L. A. N. Amaral, Eur. Phys. J. B 38, 381 (2004).

[15] M. -H. Li, Y. Fan, J. -W. Chen, L. Gao, Z. -R. Di and J. -S. Wu, Physica A 350, 643 (2005).

[16] P. -P. Zhang, K. Chen, Y. He, T. Zhou, B. -B. Su, Y. -D. Jin, H. Chang, Y. -P. Zhou, L. -C. Sun, B. -H. Wang and D. -R. He, Physica A 360, 599 (2005).

[17] A. Barrat, M. Barthélemy, R. Pastor-Satorras and A. Vespignani, Proc. Natl. Acad. Sci. U.S.A. 101, 3747 (2004).

[18] A. Barrat, M. Barthélemy and A. Vespignani, Phys. Rev. 
Lett. 92, 228701 (2004).

[19] A. Barrat, M. Barthélemy and A. Vespignani, Phys. Rev. E 70, 066149 (2004).

[20] A. Vázquez, Phys. Rev. E 67, 056104 (2003).

[21] R. Xulvi-Brunet and I. M. Sokolov, Phys. Rev. E 70, 066102 (2004).

[22] M. Catanzaro, G. Caldarelli and L. Pietronero, Phys. Rev. E 70, 037101 (2004).

[23] W. -X. Wang, B. Hu, T. Zhou, B. -H. Wang and Y. -B. Xie, Phys. Rev. E 72, 046140 (2005).

[24] W. -X. Wang, B. -H. Wang, B. Hu, G. Yan and Q. Ou, Phys. Rev. Lett. 94, 188702 (2005).

[25] J. -G. Liu, Y. -Z. Dang, W. -X. Wang, Z. -T. Wang, T. Zhou, B. -H. Wang, Q. Guo, Z. -G. Xuan, S. -H. Jiang and M. -W. Zhao, arXiv:physics/0512270
[26] J. -G. Liu, Y. -Z. Dang and Z. -T. Wang, Physica A 366, 578 (2006).

[27] S. Wasserman and K. Fast, Social Networks Analysis, (Cabridge University Press, Cambridge, 2001).

[28] J. Scott, Social Network Analysis: A Handbook, (2nd ed., Sage Publications, London, 2000).

[29] M. E. J. Newman, Phys. Rev. Lett. 89, 208701 (2002).

[30] M. E. J. Newman, J. Park, Phys. Rev. E 68, 036122 (2003).

[31] C. L. Freeman, Sociometry, 40, 35 (1977).

[32] L. C. Freeman, Social Networks, 1, 215 (1979).

[33] M. E. J. Newman, M. Girvan, Phys. Rev. E 69, 026113 (2004). 


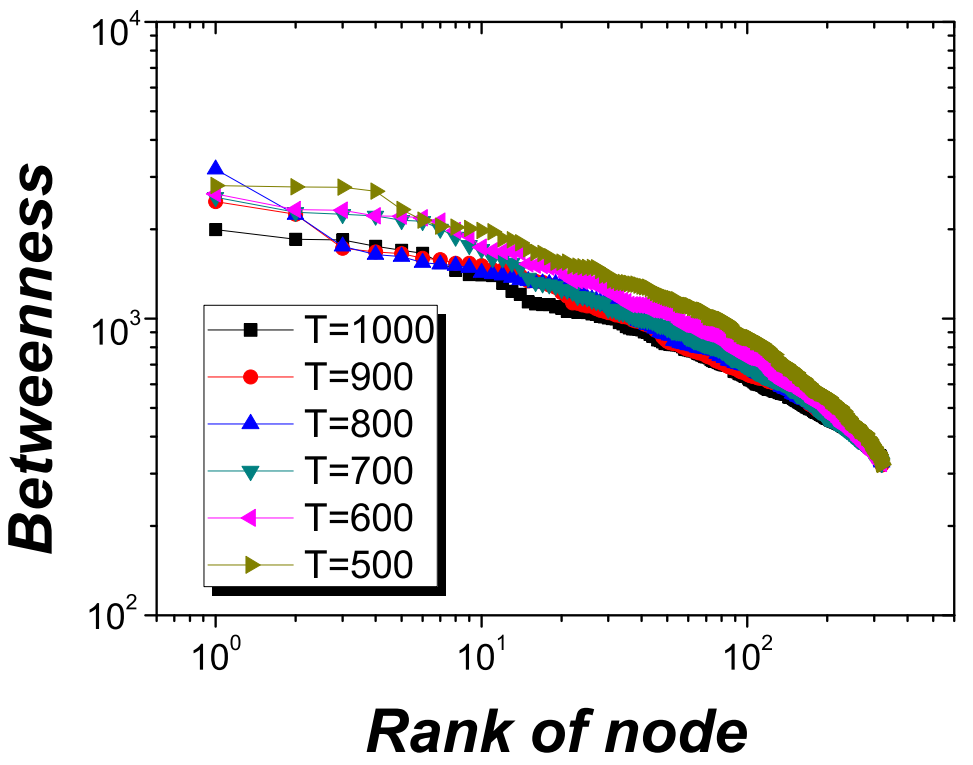

